\title{
Wave attenuation in a marginal ice zone due to the bottom roughness of ice floes
}

\author{
Alison L. KOHOUT, ${ }^{1}$ Michael H. MEYLAN, ${ }^{2}$ David R. PLEW ${ }^{1}$ \\ ${ }^{1}$ National Institute of Water and Atmospheric Research Ltd, PO Box 8602, Christchurch, New Zealand \\ E-mail: a.kohout@niwa.co.nz \\ ${ }^{2}$ Department of Mathematics, University of Auckland, Private Bag 92019, Auckland, New Zealand
}

\begin{abstract}
Wave attenuation in a diffuse marginal ice zone (MIZ) is thought to be mainly a result of wave scattering. In a compact MIZ, additional physical factors are thought to be relevant. In this paper, we propose that viscous drag, form drag and energy lost to internal waves under the ice play a role in attenuating wave energy. We derive a relation for the wave attenuation due to drag. We combine the drag attenuation coefficient with the scattering attenuation coefficient and compare the result to experimental results for compact MIZs. We find that the combined scatter and drag (CSD) model improves the rate of decay of wave attenuation in compact ice fields, but fails to predict the 'rollover' seen at short periods.
\end{abstract}

\section{INTRODUCTION}

The presence of sea ice in the polar regions plays an important role in the world's climatic system, and it is therefore important to understand the processes influencing the formation and deformation of sea ice. Ocean waves play a major role in the fracturing of ice floes in the polar regions (Squire and others, 1995; Wadhams, 2000). In order to understand this process, it is critical to understand the attenuation of waves as they enter the ice-covered seas. Wave and ice interaction is a complex phenomenon and the processes responsible for attenuating the wave energy are not fully understood. For a marginal ice zone (MIZ) consisting of discrete solitary floes, it has been assumed that the scattering of wave energy by individual ice floes is the dominant factor attenuating wave energy (Squire and others, 1995). Kohout and Meylan (2008) present a wave-scattering model based on the elastic bending of ice floes. They compare the model to observed data from diffuse MIZs and find it successfully predicts the attenuation of wave energy. They also compare their wave-scattering model to observed data from compact MIZs, but find it underestimates the wave attenuation. Presumably this is a result of a reduced presence of individual floes scattering the wave energy. Shen and Squire (1998) investigate additional factors which may contribute to wave attenuation in sea ice. These include wave-energy absorption due to hysteresis as floes deform on the passing wave field, absorption in the water column from processes such as wave breaking and absorption due to collisions and other interactions between floes.

Liu and Mollo-Christensen (1988) describe a model for wave decay, which assumes attenuation is due to the viscous boundary layer under ice. It is assumed that an oscillating boundary layer develops under the ice, causing energy loss. They parameterize the energy loss by a tuning parameter, the eddy viscosity, which is related to actual flow conditions. Their model agrees well with experimental data. A further summary of MIZ experiments and models is presented by Squire (2007).

Here we extend the scattering model of Kohout and Meylan (2008) by considering the bottom roughness of ice floes.

\section{WAVE SCATTERING AND MIZ SIMULATION}

We simulate the scattering behaviour of waves in a MIZ using the technique described by Kohout and Meylan (2008). The problem consists of a set of floes of finite length bounded by a semi-infinite floe at either end. The standard mathematical assumptions of the problem hold, i.e. throughout the fluid Laplace's equation holds, the seabed is impermeable so the velocity component normal to the sea-floor vanishes, the seabed is at a constant depth, the thin elastic plate equation holds at the surface, and the submergence of the floes is considered negligible. We further assume that the problem is invariant in the $y$ direction, the direction parallel to the ice edge, and, for simplicity, allow the waves to be normally incident. The floe edges are assumed free to move at each floe boundary. Each floe is defined independently, and open-water sections are simulated by defining the section as a very thin floe. This technique is chosen to avoid introducing an additional formulation. A schematic diagram of the problem is shown in Figure 1.

An eigenfunction expansion is used to solve the problem. The velocity potential under the floes is expressed as a summation of the reflected and transmitted travelling, damped and evanescent waves (Fig. 1). The coefficients in the expansion are solved by applying free boundary conditions and matching the potential and its $x$ derivative at each floe boundary.

This model is used to simulate an ocean wave propagating under a set of ice floes. We set the water depth sufficiently large that it can be considered infinite and set the number of evanescent modes to 20, which is a good compromise between accuracy and computational effort. In all results presented, the semi-infinite floe on the left is open water and the second floe represents the ice edge. To simulate the MIZ, gaps of water between each floe are added and the thickness of each floe is chosen normally about a mean. A wave is incident from the lefthand (open-water) side with unit amplitude, and we use the following variables: incident period, $T$; floe length, $L$; floe concentration, $C$; and number of floes, $\Lambda$. Note that the floe length and floe thickness can differ from floe to floe. 


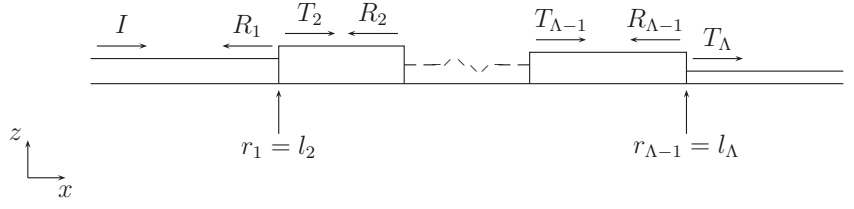

Fig. 1. A schematic diagram showing the set of ice floes and the coordinate systems used in the solution. The two-dimensional region is defined by $-\infty<x<\infty$ and $-h<z \leq 0$, where we assume constant depth, $h . l$ is the incident wave. $R_{\mu}$ and $T_{\mu}$ are the reflection and transmission coefficients of the $\mu$ th floe. $I_{\mu}$ and $r_{\mu}$ are the left and right edges of floe $\mu$. There are $\Lambda$ floes, where the first and last are semi-infinite. Note that the first floe represents the open ocean.

\section{WAVE ATTENUATION}

Experiments have shown that wave energy, $E$, decays approximately exponentially with distance, $x$, into an icecovered sea (Wadhams, 1978). Hence, for a given $E$ and $x$, an attenuation coefficient, $\alpha$, can be calculated such that

$$
E=\mathrm{e}^{\alpha x} \text {. }
$$

Experiments have also shown that short waves are attenuated quickly (increased $\alpha$ ) and long waves can propagate further into the ice (decreased $\alpha$ ). Experimental data also show a 'rollover' phenomenon, where at a critical period, varying between 4 and $8 \mathrm{~s}$, the attenuation coefficient peaks. Prior to the peak, the attenuation coefficient increases with increasing period (Figs 2-4). Squire and Wadhams (1985) suggest that the 'rollover' may be explained by an increase in energy at short periods due to local generation of waves and wind. The location of the 'rollover' depends on ice conditions, especially ice thickness (Liu and others, 1991).

\section{Scattering}

The condition that the sum of the radiated energy must be equal to the incident wave energy implies that the transmitted

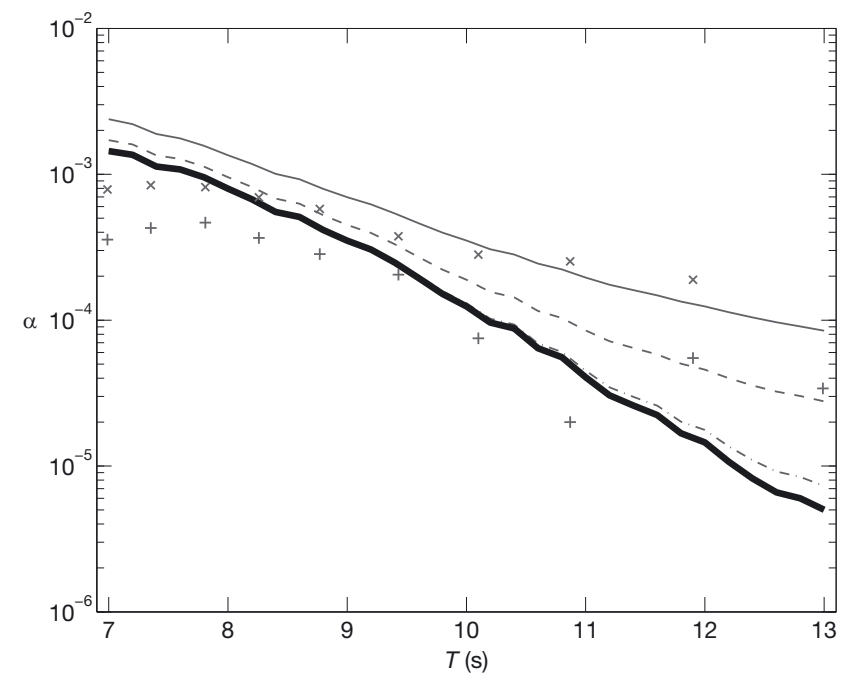

Fig. 2. Bering Sea observations from the two runs on 7 February 1983 $(x$ and +$)$. The attenuation coefficients, $\alpha$, from the scatter model (thick solid curve) and the combined scatter and drag (CSD) model with $C_{\mathrm{d}}=0.001$ (dot-dashed curve), $C_{\mathrm{d}}=0.01$ (dashed curve) and $C_{\mathrm{d}}=0.035$ (thin solid curve) against wave period, $T$. Each model calculates the attenuation assuming $C=0.72$ and $L=14.5 \mathrm{~m}$.

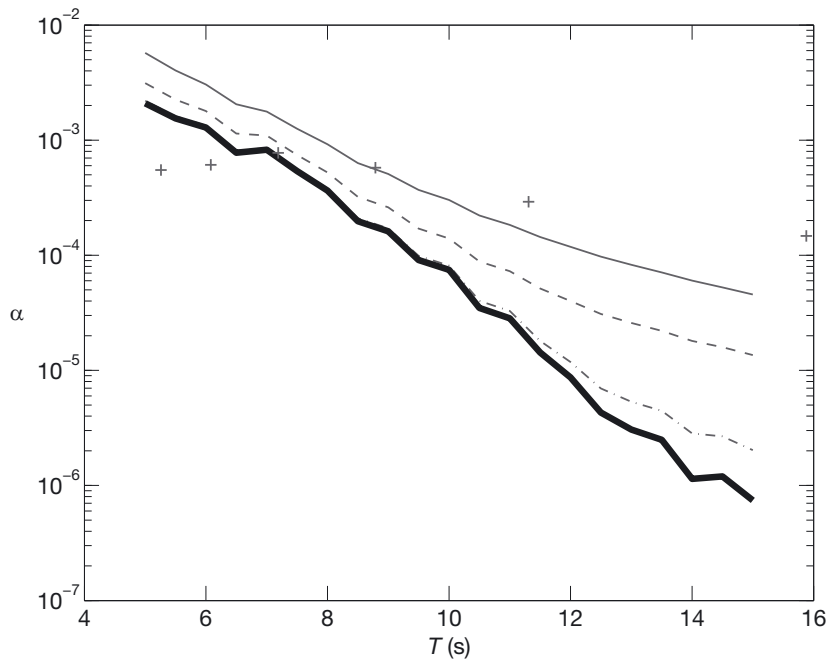

Fig. 3. Bellingshausen Sea observations from run $323(+)$. The attenuation coefficients, $\alpha$, from the scatter model (thick solid curve) and the CSD model with $C_{\mathrm{d}}=0.001$ (dot-dashed curve), $C_{\mathrm{d}}=0.01$ (dashed curve) and $C_{\mathrm{d}}=0.035$ (thin solid curve) against wave period, $T$. Each model calculates the attenuation assuming $C=0.6$ and $L=20 \mathrm{~m}$.

energy in the right-hand semi-infinite floe, $E$, can be defined as $E=1-\left|R_{1}\right|^{2}$, where $\left|R_{1}\right|$ is the reflection coefficient of the first floe. In the scattering model of Kohout and Meylan (2008), wave energy decays exponentially with increasing number of floes, $\Lambda$, i.e.

$$
E \propto \mathrm{e}^{-\alpha_{\mathrm{s}} \Lambda}
$$

where $\alpha_{\mathrm{s}}$ is the attenuation coefficient due to wave scattering. The scattering model is used to estimate $E$ for a given number of floes. We express the exponential curve as a linear function by taking the natural logarithm of both sides, i.e. $\ln E=-\alpha_{\mathrm{s}} \Lambda$. A straight line is fitted to the model output and

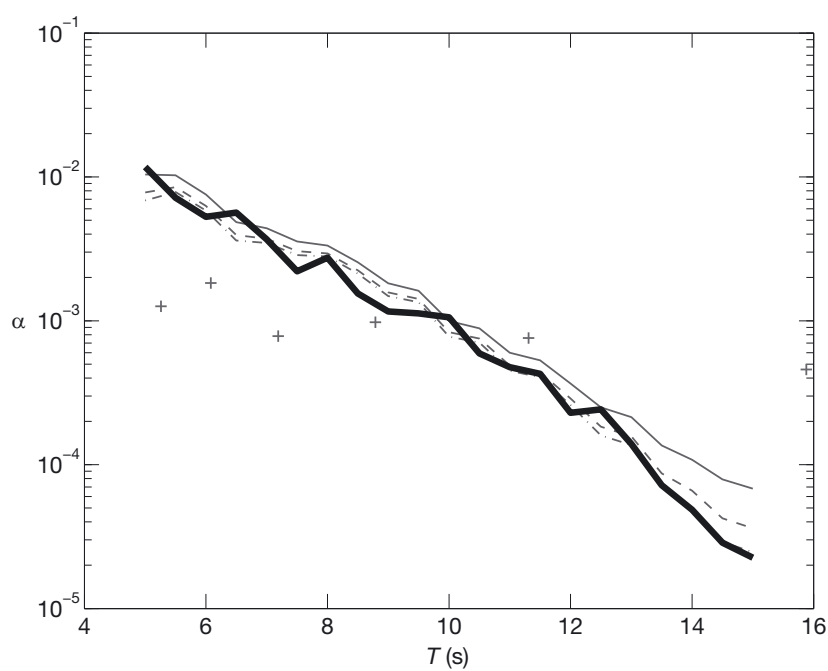

Fig. 4. Bellingshausen Sea observations from run 324 (+). The attenuation coefficients, $\alpha$, from the scatter model (thick solid curve) and the CSD model with $C_{\mathrm{d}}=0.001$ (dot-dashed curve), $C_{\mathrm{d}}=0.01$ (dashed curve) and $C_{\mathrm{d}}=0.035$ (thin solid curve) against wave period, $T$. Each model calculates the attenuation assuming $C=0.8$ and $L=20 \mathrm{~m}$. 
we solve for $\alpha_{\mathrm{s}}$ by minimizing the sum of squares of errors between the model output and the linear curve.

Kohout and Meylan (2008) show that the approximation for $\alpha_{\mathrm{S}}$ does not predict the 'rollover' and hence consistently over-predicts the attenuation of short-period waves.

S. Muzylev (personal communication 2010) suggests the model misses the 'rollover' because of the fundamental irrotational assumption of the fluid, which is a necessary assumption for Laplace's equations to hold. For periods greater than the 'rollover' tipping point, the scattering model is generally a good approximation for the attenuation of waves in a diffuse MIZ. In a compact MIZ, however, the attenuation for long periods is much greater than can be accounted for by wave scattering. We know this from both measurements and theory (Kohout and Meylan, 2008). This is actually what we would expect in a compact ice field as there are fewer ice edges present to scatter the wave energy. We do not currently know for certain what physical process is causing this attenuation for the long periods and, in the next subsection, we investigate the effects of drag as a possible cause.

\section{Drag}

It is thought that both skin friction and form drag are relevant forms of drag under sea ice, but the separation of the two forms of drag is not well understood (McPhee and others, 1987; McPhee and Kantha, 1989). When the ocean is densely covered by ice floes, i.e. when ice concentrations are high, the total stress on the underside of sea ice is thought to mainly comprise turbulent skin friction (Steele and others, 1989). However, form drag across pressure-ridge keels and other protuberances may also play a significant role (McPhee and Kantha, 1989). Further, when concentrations are $<100 \%$, form drag due to the flow of water against the edge of an ice floe may become more important than tangential skin friction drag (Steele and others, 1989). Steele and others (1989) suggest that, since both form drag and skin friction are proportional to the difference of ice and water velocities squared, times a characteristic area (the surface area for skin friction and the frontal area for form drag), the drag coefficient for skin friction is $\sim 1 / 300$ times that for form drag. Steele and others (1989) conclude that form drag becomes more important than skin friction for floes with diameters approximately 300 times less than the ice draft. Such floes are characteristic of floes typically found in a MIZ.

Internal wave drag further complicates matters (McPhee and Kantha, 1989). A series of stratified tow-tank experiments, documented by Carstens and McClimans (1980) and Muench and Hachmeister (1984), show that under conditions similar to those in a MIZ with ridged keels, internal wave drag could be just as significant as form drag (McPhee and Kantha, 1989).

Measuring drag under sea ice is a challenging and expensive task. Despite this, extensive measurements of drag were conducted under drifting ice in the Beaufort Sea during the Arctic Ice Dynamics Joint Experiment (AIDJEX) (Hunkins, 1974; McPhee and Smith, 1976; Langleben, 1980). Of particular relevance are the experiments during the MIZ experiment (MIZEX), which made a considerable contribution to knowledge of upper-layer processes under first-year sea ice in MIZs (Shirasawa and Ingram, 1991). In general, observations and estimates of the ice/water drag coefficient have shown that it can vary between $1 \times 10^{-3}$ and $35 \times 10^{-3}$ (Morison and others, 1987; Shirasawa and
Ingram, 1991). Steele and others (1989) suggest that the coefficient is dependent on ice type, ice draft, floe size and ice concentration.

Here we derive an expression for the attenuation of wave energy in terms of a drag coefficient. Assuming steady flow and also that inertial acceleration is insignificant, shear stress from water flowing past a surface can be defined as a function of the drag coefficient, $C_{\mathrm{d}}$,

$$
\tau=\rho C_{\mathrm{d}} u^{2},
$$

where $\tau$ is the stress, $\rho$ is the density of the fluid and $u$ is the relative velocity of water to ice (Langleben, 1982). We assume the ice floe has minimal movement and use the standard definition for fluid velocity in deep water,

$$
u=\frac{1}{2} \omega H \mathrm{e}^{\frac{2 \pi z}{\lambda}} \cos \theta,
$$

where $H$ is wave height, $\omega$ is wave frequency, $z$ is water depth, $\lambda$ is wavelength and $\theta=\omega t+k x$ where $k$ is the wavenumber, $t$ is time and $x$ is the distance of propagation (US Army Corps of Engineers, 2002). Here we consider the drag at the surface and take $z=0$. We assume maximum velocity, so $\cos \theta=1$. Hence, $u$ simplifies to

$$
u=\frac{1}{2} \omega H \text {. }
$$

The power dissipation per unit area is defined as

$$
P=\tau u,
$$

which can also be described as the decay of energy with respect to time or

$$
\frac{\mathrm{d} E}{\mathrm{~d} t}=\frac{1}{8} \rho C_{\mathrm{d}} \omega^{3} H^{3}
$$

The decay of wave energy can also be represented by

$$
\frac{\mathrm{d} E}{\mathrm{~d} t}=\frac{-2 \rho g H}{8} \frac{\partial H}{\partial t}
$$

given that $\mathrm{d} E / \mathrm{d} t=(\partial E / \partial H)(\partial H / \partial t)$ and $E=\rho g H^{2} / 8$. Combining Equations (7) and (8) and simplifying gives

$$
\frac{1}{H^{2}} \frac{\partial H}{\partial t}=-\frac{1}{2} C_{\mathrm{d}} k \omega
$$

since $\omega^{2}=k g$ for deep water. Integrating gives

$$
\int_{H_{0}}^{H} \frac{1}{H^{2}} \mathrm{~d} H=-\int_{0}^{t} \frac{1}{2} C_{\mathrm{d}} k \omega \mathrm{d} t
$$

where $H_{0}$ is the initial wave height. Evaluating gives

$$
\frac{1}{H}=\frac{1}{H_{0}}+\frac{1}{2} C_{\mathrm{d}} k \omega t
$$

Rearranging and substituting $t=x / C_{\mathrm{g}}$ and $C_{\mathrm{g}}=g / 2 \omega$, where $C_{\mathrm{g}}$ is the group speed, gives

$$
H=\frac{H_{0}}{1+C_{d} k^{2} H_{0} x}=f(x) .
$$

This gives an expression for the wave height as a function of distance, $x$. The decay is not exponential because the effect of drag is nonlinear. However, the decay can be approximated as exponential over short distances, i.e. we can approximate the scattering as linear over short distances as long as only a fraction of the energy is dissipated. We 
write the energy as (where the constant, $B$, relating energy to wave height, does not appear in the final formula)

$$
E=B H^{2}=B\left(\frac{H_{0}}{1+C_{\mathrm{d}} k^{2} H_{0} x}\right)^{2}=B(f(x))^{2} .
$$

If the decay of energy was exponential (which it is not) then $E=E_{0} \mathrm{e}^{-\alpha_{\mathrm{d}} x}$, so

$$
\frac{\mathrm{d} E}{\mathrm{~d} x}=-\alpha_{\mathrm{d}} E
$$

Therefore, we can approximate the decay as a function of $x$ as

$$
\frac{\mathrm{d} E}{\mathrm{~d} x}=2 B f^{\prime}(x) f(x)=2 \frac{f^{\prime}(x)}{f(x)} E(x),
$$

so that the decay constant, $\alpha_{\mathrm{d}}$, is given by

$$
\alpha_{\mathrm{d}}=-2 \frac{f^{\prime}(x)}{f(x)}=-\frac{2}{H} \frac{\mathrm{d} H}{\mathrm{~d} x} .
$$

We also know that

$$
\frac{1}{H}-\frac{1}{H_{0}}=C_{\mathrm{d}} k^{2} x
$$

so

$$
x=\frac{1}{C_{\mathrm{d}} k^{2}}\left(\frac{1}{H}-\frac{1}{H_{0}}\right)
$$

and therefore

$$
\frac{\mathrm{d} x}{\mathrm{~d} H}=-\frac{1}{C_{\mathrm{d}} k^{2} H^{2}} \text {. }
$$

Therefore

$$
\alpha_{\mathrm{d}}=2 H C_{\mathrm{d}} k^{2} .
$$

Note that the decay rate is linearly proportional to wave height.

We combine the drag attenuation coefficient, $\alpha_{\mathrm{d}}$, with the wave-scattering attenuation coefficient, $\alpha_{s}$, to estimate a combined attenuation coefficient, $\alpha$. The scattering attenuation coefficients, $\alpha_{\mathrm{s}}$, are per floe number $(\Lambda=x C / L$, where $L$ is the average length of the floes and $C$ is the floe concentration). We now define the combined attenuation coefficient as a function of distance

$$
\alpha=\frac{C}{L} \alpha_{\mathrm{s}}+\alpha_{\mathrm{d}}
$$

\section{COMPARISONS BETWEEN MODEL AND OBSERVATIONS}

A valuable set of observations measuring wave attenuation in a MIZ was collected by the Scott Polar Research Institute (SPRI) in the late 1970s and early 1980s (Squire and Moore, 1980; Squire and others, 1983; Wadhams and others, 1988). Wadhams and others (1988) give the attenuation coefficients derived from observations in compact ice from the Bering Sea in February 1983. More recently, a set of observations in compact ice was gathered using an autonomous underwater vehicle (AUV) in the western Bellingshausen Sea (Hayes and Jenkins, 2007). The Autosub AUV completed two successful runs (Belling 323 and 324) west of the Antarctic Peninsula during 22-25 March 2003. A detailed description of these observations and a comparison to the scattering model is given by Kohout and Meylan (2008).

Here we compare the energy attenuation coefficients calculated from the SPRI and AUV experimental data against the scatter model and the combined scatter and drag (CSD) model.
The Bering Sea 1983 experiments are presented in Figure 2 (note there were two experiments on the same day with different results, despite the conditions being almost identical). As discussed in the previous section, the scatter model overestimates the attenuation for short periods and underestimates for long periods. Note that with an ice concentration of 0.72 , this experiment lies in the transition between diffuse and compact. Compared to the scatter model, the CSD model decays slower with increasing period, but simultaneously increases the estimate of the attenuation at shorter periods and hence accentuates the misrepresentation of attenuation at periods relating to the 'rollover'.

The Autosub AUV data are shown in Figures 3 and 4. Note that here we leave out the $26 \mathrm{~s}$ wave period observations of Hayes and Jenkins (2007). This is because, as Hayes and Jenkins (2007) noted, the attenuation coefficients for waves of periods $>16 \mathrm{~s}$ might be compromised by measuring capabilities and possible surge responses of the vehicle. For the Belling 323 experiment, the scattering model again misrepresents the 'rollover' at short periods and significantly underestimates the wave attenuation at longer periods (Fig. 3). The CSD model improves the rate of decay of the attenuation coefficient, but again accentuates the misrepresentation at periods relating to the 'rollover'. The CSD model for the Belling 324 experiment does not significantly differ from the scatter model (Fig. 4). During the Belling 324 experiment, the ice was $\sim 2$ m thick, which increases attenuation due to scattering, so attenuation due to drag becomes relatively insignificant.

The addition of drag to the scattering model does improve the rate of decay of the attenuation coefficient, but simultaneously weakens the approximation for periods relating to the 'rollover'. Strictly speaking, a pure scattering model will never correctly predict the 'rollover' effect and either a parameterization or a physical representation of this phenomenon should be included. Another oversimplification lies in the choice of a constant drag coefficient. In reality, the drag coefficient will change with distance into an ice field, i.e. older ice may be more heavily rafted than newer ice which forms at the ice/ocean boundary. Further, the drag coefficient will vary with ice concentration, which is also likely to change with distance into the ice, generally becoming more compact with distance into the ice field. It is possible to include other parameterizations in the wave-attenuation model, including viscous losses within the ice floes, collisional and floe-breaking losses. The problem lies in difficulties associated with measuring these parameters and currently there is not enough knowledge to even approximate these parameters. To accurately represent wave attenuation in a MIZ, parameter tuning with sensitivity tests will be necessary in the future.

\section{SUMMARY}

To summarize, wave attenuation in a diffuse MIZ is largely characterized by wave scattering (excluding short periods where a 'rollover' effect takes place). In a compact MIZ, additional physical factors are thought to be relevant. Here we have proposed that viscous, form and internal wave drag under the ice also play a role in attenuating wave energy. We have derived a relation for wave attenuation due to drag and approximated it as an exponentially decaying function. We combined the drag attenuation 
coefficient with the scattering attenuation coefficient and have compared the results against experiment results for compact MIZs. We find that the CSD model improves the rate of decay of the attenuation coefficient with increasing period, but simultaneously weakens the representation of the attenuation at the 'rollover'. Neither the scatter model nor the drag model, however, fully accounts for the damping at long periods or the 'rollover' seen at short periods, and it is thought that a parameterization of additional physical processes may be required to accurately model wave attenuation in a MIZ.

\section{ACKNOWLEDGEMENTS}

We thank the Scientific Editor and the reviewers for valuable feedback. This research was supported by New Zealand's Foundation of Research, Science and Technology and the National Institute of Water and Atmospheric Research.

\section{REFERENCES}

Carstens, T. and T. McClimans. 1980. Laboratory studies of stratified flow interaction with topography. In Carstens, T. and T. McClimans, eds. Proceedings of the 2nd International Symposium on Stratified Flows, June 1980, Trondheim, Norway. Vol. 2. Trondheim, Tapir, 623-635.

Hayes, D.R. and A. Jenkins. 2007. Autonomous underwater vehicle measurements of surface wave decay and directional spectra in the marginal sea ice zone. J. Phys. Oceanogr., 37(1), 71-83.

Hunkins, K. 1974. An estimate of internal wave drag on pack ice. AIDJEX Bull., 26, 141-152.

Kohout, A.L. and M.H. Meylan. 2008. An elastic plate model for wave attenuation and ice floe breaking in the marginal ice zone. J. Geophys. Res., 113(C9), C09016. (10.1029/2007JC004434.)

Langleben, M.P. 1980. Water drag coefficient at AIDJEX, Station Caribou. IAHS Publ. 124 (Symposium at Seattle 1977 - Sea Ice Processes and Models), 464-471.

Langleben, M.P. 1982. Water drag coefficient of first-year sea ice. J. Geophys. Res., 87(C1), 573-578.

Liu, A.K. and E. Mollo-Christensen. 1988. Wave propagation in a solid ice pack. J. Phys. Oceanogr., 18(11), 1720-1712.

Liu, A.K., B. Holt and P.W. Vachon. 1991. Wave propagation in the marginal ice zone: model predictions and comparisons with buoy and synthetic aperture radar data. J. Geophys. Res., 96(C3), 4605-4621.

McPhee, M.G. and L.H. Kantha. 1989. Generation of internal waves by sea ice. J. Geophys. Res., 94(C3), 3287-3302.
McPhee, M.G. and J.D. Smith. 1976. Measurements of the turbulent boundary layer under pack ice. J. Phys. Oceanogr., 6(5), 696-711.

McPhee, M.G., G.A. Maykut and J.H. Morison. 1987. Dynamics and thermodynamics of the ice/upper ocean system in the marginal ice zone of the Greenland Sea. J. Geophys. Res., 92(C7), 7017-7031.

Morison, J.H., M.G. McPhee and G.A. Maykut. 1987. Boundary layer, upper ocean, and ice observations in the Greenland Sea marginal ice zone. J. Geophys. Res., 92(C7), 6987-7011.

Muench, R.D. and L.E. Hachmeister. 1984. Internal wave forces on ice keels in the marginal ice zone: some preliminary laboratory results. CRREL Spec. Rep. 84-7, 83-90.

Shen, H.H. and V.A. Squire. 1998. Wave damping in compact pancake ice fields due to interactions between pancakes. In Jeffries, M.O., ed. Antarctic sea ice: physical processes, interactions and variability. Washington, DC, American Geophysical Union, 325-341.

Shirasawa, K. and R.G. Ingram. 1991. Characteristics of the turbulent oceanic boundary layer under sea ice. Part 1: a review of the ice-ocean boundary layer. J. Mar. Syst., 2(1), 153-160.

Squire, V.A. 2007. Of ocean waves and sea-ice revisited. Cold Reg. Sci. Technol., 49(2), 110-133.

Squire, V.A. and S.C. Moore. 1980. Direct measurement of the attenuation of ocean waves by pack ice. Nature, 283(5745), 365-368.

Squire, V.A. and P. Wadhams, 1985. Some wave attenuation results from MIZEX-West. CRREL Spec. Rep. 85-06.

Squire, V.A., P. Wadhams, A.M. Cowan, S.P. O'Farrell and R. Weintraub. 1983. MIZEX 83 data summary. Cambridge, Scott Polar Research Institute. (Tech. Rep. 83-1.)

Squire, V.A., J.P. Dugan, P. Wadhams, P.J. Rottier and A.K. Liu. 1995. Of ocean waves and sea ice. Annu. Rev. Fluid Mech., 27, $115-168$

Steele, M., J.H. Morison and N. Untersteiner. 1989. The partition of air-ice-ocean momentum exchange as a function of ice concentration, floe size and draft. J. Geophys. Res., 94(C9), 12,739-12,750.

US Army Corps of Engineers. 2002. Coastal engineering manual. Washington, DC, US Army Corps of Engineers. 1110-2-1100 (Tech. Rep. EM).

Wadhams, P. 1978. Wave decay in the marginal ice zone measured from a submarine. Deep-Sea Res., 25, 23-40.

Wadhams, P. 2000. Ice in the ocean. Amsterdam, etc., Gordon and Breach.

Wadhams, P., V.A. Squire, D.J. Goodman, A.M. Cowan and S.C. Moore. 1988. The attenuation rates of ocean waves in the marginal ice zone. J. Geophys. Res., 93(C6), 6799-6818. 